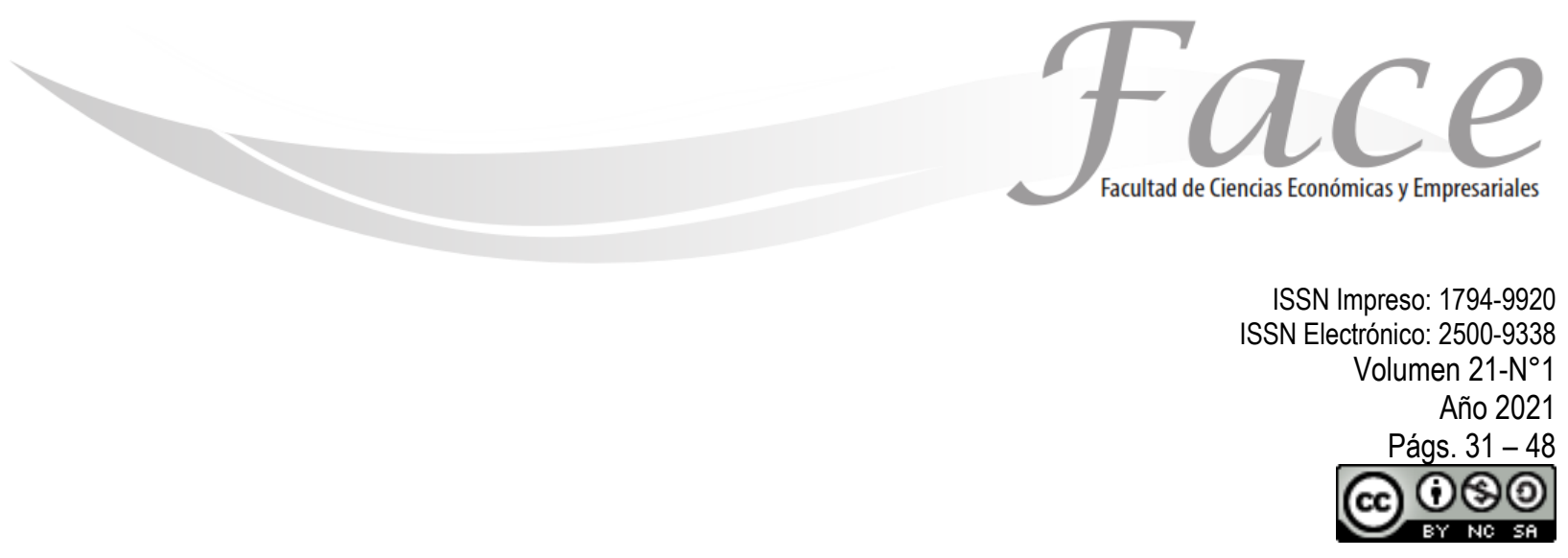

\title{
HÁBITOS DE COMPRA DE ALIMENTOS DE LAS FAMILIAS RESIDENTES EN LA ZONA DE VILLA CAMPESTRE DE BARRANQUILLA, COLOMBIA
}

\author{
Carlos Andrés Ortiz Coronado * \\ Enlace ORCID: https://orcid.org/0000-0003-4007-0487 \\ Dagoberto Páramo Morales * * \\ Enlace ORCID: https://orcid.org/0000-0002-2044-6179
}

Fecha de Recepción: Abril 4 de 2021

Fecha de Aprobación: Julio 13 de 2021

\section{Resumen:}

Esta investigación presenta los resultados de un estudio relacionado con los hábitos de compra de los consumidores de alimentos básicos de los residentes de la zona de Villa Campestre en Puerto Colombia -Atlántico- que como zona semiurbana (ZSU) ha venido creciendo de manera sostenida en los últimos años. El problema de investigación fue abordado a través de un enfoque cualitativo, particularmente con la rigurosidad y los fundamentos de la "teoría fundada". Los resultados muestran aspectos relacionados con las decisiones de consumo, preferencias por determinados locales comerciales, y una tipología de consumidores basados en quienes hacen la compra, así como por el tipo de local comercial más utilizado.

Palabras clave: Hábitos de compra, Comportamiento del consumidor, canales de compra.

\footnotetext{
* Magister en Administración de Empresas. Universidad del Norte. Especialista en Sistemas de Telecomunicaciones. Universidad del Norte. Ingeniero Electrónico. Universidad del Norte. Contacto: caortiz@uninorte.edu.co

* * Profesor e Phd. en Ciencias Económicas y Sociales, Universidad de Ginebra, Suiza. Magister en Administración, Universidad de Tulane, USA. Magister en Administración y Gestión, Universidad Católica de Lovaina, Bélgica. Profesional en Marketing, Universidad de Ejecutivos de Ventas y Mercadotecnia, México. Profesor de marketing. Universidad del Norte, Colombia. Contacto: dparamo@uninorte.edu.co
} 


\title{
FOOD BUYING HABITS OF FAMILIES LIVING IN THE VILLA CAMPESTRE AREA OF BARRANQUILLA, COLÔMBIA
}

\begin{abstract}
:
This research presents results of a study related to purchasing habits of consumers of basic foods of Villa Campestre area residents in Puerto Colombia -Atlántico- which as a semi-urban area (ZSU) has been growing steadily in recent years. The research problem was approached through a qualitative approach, particularly with the rigor and fundamentals of "grounded theory". The results show aspects related to consumption decisions, preferences for certain commercial premises, and a typology of consumers based on who makes the purchase, as well as the type of commercial premises most used.
\end{abstract}

Keywords: Shopping habits, consumer behavior, shopping channels.

\section{HÁBITOS DE COMPRA DE ALIMENTOS DAS FAMÍLIAS QUE VIVEM NA ÁREA DE VILLA CAMPESTRE DE BARRANQUILLA, COLÔMBIA}

Resumo:

Esta investigação apresenta os resultados de um estudo relacionado com os hábitos de compra dos consumidores de alimentos básicos dos residentes da zona de Villa Campestre em Puerto Colombia -Atlántico- que como zona semiurbana (ZSU) tem vindo a crescer de forma constante nos últimos anos. O problema da investigação foi abordado através de uma abordagem qualitativa, particularmente com o rigor e os fundamentos da "teoria fundamentada". Os resultados mostram aspectos relacionados com decisões de consumo, preferências para certos pontos de venda a retalho, e uma tipologia de consumidores baseada em quem faz a compra, bem como pelo tipo de ponto de venda a retalho mais utilizado.

Palavras-chave: hábitos de compra, comportamento do consumidor, canais de compra. 


\section{INTRODUCCIÓN:}

En los últimos años el perfil del consumidor colombiano respecto a sus hábitos de compra ha ido cambiando a causa de múltiples factores entre los que se destacan los avances tecnológicos y de comunicaciones, el arribo y posicionamiento de las tiendas de descuento (Hard Discount), y, el cambio y aparición de nuevos canales de compra como los digitales.

Esta investigación realizó un estudio de comportamiento de consumidor con respecto a los hábitos de compra de los productos del mercado básico. La zona en la que se desarrolló la investigación fue la de Villa Campestre, que aunque hace parte del municipio de Puerto Colombia, está muy relacionado con la ciudad de Barranquilla debido a su cercanía geográfica y emocional; está en sus alrededores. Esta zona es de especial interés debido al clima de dinamismo y crecimiento que se percibe. Esta área sigue una tendencia que se vive en muchas de las grandes ciudades del país desde hace varios años en las cuales muchas familias decidieron invertir en viviendas en su periferia, en su decisión de alejarse del bullicio que se genera en su interior. Desde hace varios años en este sector se observa un crecimiento acelerado de vivienda familiar conformada en su gran mayoría por proyectos habitacionales de varias torres de gran altura y múltiples conjuntos de casas residenciales, en estratos 3, 4 y 5 . Debido a este crecimiento residencial, han surgido varias zonas de locales comerciales (algunos con mayor éxito que otros) con múltiples servicios como restaurantes, iglesias, colegios, etc. Con notable aumento de la densidad de población, varios de los más conocidos supermercados del país decidieron hacer presencia en la zona, destacándose las llamadas tiendas de descuento (Hard Discount). Así, en esta zona se encuentran 2 Supermercados Carulla, 2 Supermercados Olímpica, una tienda Ara, una tienda D1, una tienda Justo y Bueno y una tienda Euro, adicionales a las decenas de tiendas de barrio y minimercados que ubicadas en el corregimiento $\mathrm{La}$ Playa, circundan la zona.

Ante este auge poblacional y comercial, la pregunta de investigación fue: ¿Cuáles son los hábitos de compra de los productos de la canasta de alimentos de las familias de la zona de Villa campestre de Barranquilla?

\section{MARCO TEÓRICO:}

Considerando que el consumidor es el punto de partida y de llegada del marketing, la trascendencia de su estudio es cada vez más determinante en las decisiones tomadas por las empresas tanto en la calidad de su oferta como en la forma de hacerla disponible, (Páramo, 2004a). Desde esta perspectiva, el abordaje del comportamiento del consumidor ha trascendido a otras esferas conceptuales y prácticas que han hecho más complejo el proceso de su comprensión. Aunque se han incorporado nuevas dimensiones -el consumo (uso) y la apropiación (identidad)- sigue teniendo un notorio impacto el de la adquisición de productos y marcas (Páramo y Ramírez, 2017). Así, se hace vital aproximarse a los aspectos, demográficos, geográficos y psicológicos, así como a sus procesos de decisión, a los factores que inciden de forma directa en tales decisiones, a las fuentes de información que sirven de referente, a las influencias situacionales $\mathrm{y}$, a los canales de compra disponibles.

\subsection{Aspectos demográficos}

Consiste en los datos estadísticos o estadísticas vitales que describen a la población, tales como: edad, sexo, ciclo de vida familiar (diversas formas que las familias pueden ir tomando con el paso del tiempo), educación, ingresos, origen étnico y otras características como la religión y la nacionalidad. Estos son de suma importancia ya que se relacionan de forma cercana con la demanda de muchos productos. Los cambios en la demografía indican el nacimiento de nuevos mercados y la eliminación de otros. (Stanton, Etzel, \& Walker, 2007).

\subsection{Aspectos geográficos.}

Para realizar un análisis de los cambios demográficos "los ejecutivos de marketing revisan los patrones actuales y las tendencias proyectadas en la distribución regional de la población para tomar decisiones que abarcan desde dónde se han de ubicar las tiendas detallistas hasta la mezcla apropiada de productos que se van a ofrecer." (Stanton, Etzel, \& Walker, 2007). Es necesario analizar la concentración poblacional o las zonas con mayor tasa de crecimiento 
entre otras. Ello implica diferenciar la población rural, la urbana, y la suburbana (ZSU).

De otro lado y como se menciona en Valencia \& López (2013) en un intento por acercarse a los estudios territoriales hechos por el University College of London a través de la Development Planning Unit, "...han condensado tres formas en las que regularmente han identificado aproximaciones a la noción de interfase, 0 ZSU en nuestro caso (Allen, da Silva, \& Corubolo, 1999). La ZSU, como la periferia de la ciudad, hace referencia a las áreas marginales en proceso de urbanización y como sistema socioeconómico, es visto como un sistema social de pobladores con una condición dual entre lo rural y lo urbano. La ZSU, como la interacción de flujos rurales y urbanos, considera las áreas periféricas de las ciudades en donde se verifican con mayor intensidad vínculos rural-urbanos y son más intensos los cambios y conflictos sociales, ambientales y económicos".

\subsection{Factores psicológicos}

Según Solomon (1997), las fuerzas psicológicas que afectan las decisiones de compra son la motivación, la percepción, el aprendizaje, la personalidad y las actitudes. La motivación se refiere a todo comportamiento resultado de una necesidad estimulada (Shiffman y Kanuk, 2001). La percepción corresponde a la forma en que se interpreta el mundo que nos rodea y que es susceptible a tres tipos de selectividad, la atención, la distorsión y la retención (Allport, 1961). El aprendizaje es un cambio en el comportamiento como resultado de la experiencia. El aprendizaje del estímulo y la respuesta comprende impulsos, claves incitativas, respuestas y reforzamiento (Páramo, 2004b). El reforzamiento positivo continuo lleva a la compra habitual y a la lealtad a la marca. La personalidad es la suma de los rasgos de un individuo que influyen en las respuestas de la conducta. Los mercadólogos, basados en la teoría psicoanalítica freudiana de la personalidad, señalan que los verdaderos motivos de la conducta a menudo están ocultos. El autoconcepto se relaciona con la personalidad (Páramo, 1999). Puesto que la compra y el consumo son acciones muy expresivas, nos permiten comunicar al mundo nuestros autoconceptos reales y el ideal. Las actitudes son predisposiciones aprendidas a responder a un objeto 0 clase de objetos de una manera uniforme (Páramo y Ramírez, 2017). Todas las actitudes se dirigen hacia un objeto, tienen dirección e intensidad, y tienden a ser estables y generalizables. Las actitudes firmemente sostenidas son difíciles de cambiar.

\subsection{Proceso de decisión de compra del consumidor}

Uno de los desafíos para los mercadólogos es entender cómo toman las decisiones los consumidores. Para ello deben mejorar su comprensión de los consumidores de manera constante $e$ intentar adaptar rápidamente sus estrategias (Solomon, 1997). "El comportamiento de compra de los consumidores finales se describe como un proceso de decisión de compra de cinco etapas influenciado por la información, las fuerzas sociales y de grupo, las fuerzas psicológicas y los factores situacionales" (Stanton, Etzel, \& Walker, 2007): 1. reconocimiento de la necesidad, 2 . identificación de alternativas, 3. evaluación de alternativas, 4. toma de decisiones, 5. comportamiento postcompra.

La experiencia ha hecho que varios autores hagan excepciones a este modelo racional que no incluye los aspectos simbólicos y emocionales recientemente descubiertos: a) el consumidor puede salirse del proceso en cualquier etapa previa a la compra real si la necesidad disminuye 0 si no hay alternativas satisfactorias disponibles, b) las etapas suelen ser de duración diferente, pueden entremezclarse y algunas pueden incluso pasarse por alto $y, c$ ) el consumidor a menudo se ve en la situación de tomar simultáneamente varias decisiones de compra diferentes, y el resultado de una puede afectar a las otras (Shiffman y Kanuk, 2001).

\subsection{Fuentes de información}

Aunque existen diversas fuentes éstas han sido clasificadas en dos: comerciales, sociales. Las fuentes comerciales están compuestas por todas las organizaciones de marketing, publicidad y personas que tratan de comunicarle a los consumidores la existencia de cada uno de los productos que deben comercializarse. Incluye a toda persona que trabaje para informar o persuadir una decisión de compra entre esos los fabricantes, detallistas, anunciantes y vendedores (Páramo y Ramírez, 2007). Otras fuentes comerciales son los dependientes de tiendas detallistas, sitios de red de negocios y vendedores telefónicos.

Las fuentes sociales se refieren a los grupos sociales que conforman la cultura, la subcultura, la clase social 
(definida como una jerarquía dentro de una sociedad determinada por sus miembros), los grupos de referencia (artistas, deportistas, influenciadores), la familia y los hogares. La cultura tiene la influencia más amplia y general en el comportamiento de compra, mientras que otros ocupantes del hogar tienen un efecto más específico e inmediato en un individuo. Las fuerzas sociales y de grupo pesan directamente en las decisiones de compra individuales, así como en la composición psicológica de la persona (Stanton, Etzel, \& Walker, 2007).

\subsection{Influencias situacionales}

De acuerdo con Stanton, Etzel \& Walker (2007), "las influencias situacionales tienen que ver con el cuándo, dónde, cómo y por qué compran los consumidores, y con la situación personal del consumidor (estado de ánimo) en el momento de la compra. Estas influencias tienen tanto peso para el comprador que pueden ser más importantes que las demás fuerzas en el proceso de decisión de compra"

Para esto se debe tener en cuenta por ejemplo la dimensión tiempo. Acá se deben responder preguntas sobre el comprador, por ejemplo, sobre ¿cómo influyen el día y la hora de la semana, la época del año en las compras? ¿Qué impacto tienen los acontecimientos presentes y pasados en la decisión de compra? ¿Cuánto tiempo tiene para hacer la compra y consumir el producto?

También se analiza el entorno físico que incluyen iluminación, olores, clima, sonidos del lugar donde realiza la compra. Así también los términos y condiciones de la venta y las actividades relacionadas con la transacción que los compradores están dispuestos a llevar a cabo (uso de tarjetas de crédito, débito, efectivo, cheques).

\subsection{Canales de compra disponibles}

Dentro de los cambios que impone el mundo moderno se encuentra también la aparición de nuevos mercados o canales de compra formados por una gran red de pequeños negocios familiares, independientes e informales, el uso de plataformas tecnológicas que permiten adquirir productos en línea, páginas web 0 aplicaciones, redes sociales con chats de respuesta inmediata que han facilitado a muchas personas la forma como realizan sus compras y que poco a poco han ido posicionándose y haciendo pensar a las empresas en la necesidad de participar de esta tendencia. La masificación de los equipos móviles, la conectividad, el acceso instantáneo a información real, confiable y actualizada han influido notablemente en los nuevos comportamientos de compra. Estos canales tienen la característica de hacerse presente en los lugares donde el cliente quiere y cuando él quiere. Son usados además de publicitar y vender un bien 0 servicio, para dar atención al cliente, entregar servicios de valor agregado y dar atención a quejas y reclamos.

Adicionalmente, estos canales mantienen la característica de poder realizar pagos de diferentes formas, a través de transferencias, depósitos, tarjetas de débito o crédito.

Una de las generaciones que más utiliza este tipo de canales son los Millenials (nacidos entre 1981 y 1999). Los miembros de esta generación serán para el 2025 más del $50 \%$ (Fujitsu, 2018) y para el 2030 , el $75 \%$ (Flores, 2016) de la fuerza laboral. Para esta generación, los productos de tecnología no son una herramienta sino una extensión de ellos mismos y reflejan la forma cómo realizan sus interacciones sociales y actividades.

Sin embargo, existen "altas barreras en la percepción de la población emergente hacia estos formatos modernos. Por ejemplo, la frecuencia de compra está determinada por la capacidad de gasto y el estilo de vida, la relevancia de la ubicación, la disponibilidad de transporte o la capacidad de almacenar los productos en casa. En este contexto se hace imprescindible que el canal moderno rompa sus paradigmas y defina un nuevo enfoque hacia un shopper que no es tan influenciable y consiga entender sus hábitos de compra y de consumo, sus preferencias y su capacidad de generación de ingresos. Pero, principalmente, se debe profundizar en la dinámica de la interacción que mantiene dicho shopper con el resiliente canal tradicional, cuya propuesta de valor está dada por factores como proximidad/conveniencia, relación lealtad-crédito y el manejar tamaños/precios accesibles." (Rodríguez, 2017). 


\section{METODOLOGÍA:}

Para la solución del problema de investigación se recurrió al enfoque cualitativo, particularmente a la rigurosidad de la teoría fundada concebida para descubrir algunos trazos teóricos (Contreras, Páramo, \& Rojano, 2019) que se ocultan tras el comportamiento humano y vista, además, como un método "para aproximarse a la realidad en circunstancias naturales recurriendo a observaciones participantes, no participantes, entrevistas en profundidad y videofilmaciones, teniendo como referente la cultura en que se desenvuelven los fenómenos cotidianos" (Páramo, García, \& Arias, 2011). Esta investigación se apoyó en la observación y descripción de los factores que precisan los hábitos de compra, y sus preferencias. Se anotaron las acciones de los entrevistados sin interacción directa. Se realizó una observación sistemática indirecta de los fenómenos y actividades desarrolladas en cada canal de venta. Lo anterior a través del análisis de los relatos obtenidos en las entrevistas en profundidad realizadas. Se elaboró una guía de entrevista que sirvió como instrumento para ordenar y estructurar la recopilación de los datos (McDaniel \& Gates, 1999).

La información que se fue recopilando fue analizada e interpretada de forma simultánea, a través de un análisis incremental. A medida que se iban realizando las entrevistas se iba agregando información hasta que se llegó a la denominada "saturación teórica" "que se alcanza cuando la información recopilada no aporta nada nuevo al desarrollo de las propiedades y dimensiones de las categorías de análisis" (Ardila \& Rueda, 2013). Se realizaron en total 11 entrevistas en profundidad de tipo semiestructurado a habitantes de la zona de Villa Campestre de diferentes orígenes sociodemográficos. Las personas eran las encargadas de realizar las compra frecuentes de los víveres de su hogar. Se descartaron personas que no fueran las directamente responsables del proceso de compra. A partir de la información consultada se procedió a elaborar un primer grupo de entrevistas con preguntas realizadas a 2 personas. Posteriormente, este instrumento fue aplicado a 9 personas más y a medida que se iba avanzando en la investigación se fueron adicionando preguntas para encontrar una representación de los perfiles de los consumidores entrevistados.
Debido a la situación de pandemia a raíz del SARSCoV-2 se realizaron entrevistas informales conversacionales a través de la plataforma Zoom. Estas entrevistas fueron grabadas con autorización del entrevistado.

Como la investigación se basó en la Grounded Theory (Teoría fundada), -manteniendo una estricta rigurosidad en la recopilación y validación de los hallazgos-, "los análisis fueron hechos a partir de los datos obtenidos de la trascripción literal de las entrevistas, de las conclusiones, de las conversaciones con expertos, de la revisión documental y de la reflexión de los investigadores" (Strauss \& Corbin, 1990).

Las entrevistas fueron sistematizadas en una matriz inicial que permitió orientar el análisis y comprensión de los fenómenos relacionados con las preferencias de compra encontradas generando diversos códigos conceptuales. (Páramo, García, \& Arias, 2011). "Para realizar la caracterización se estructuraron matrices de análisis -preguntas orientadoras generales $y$ preguntas específicas- en las que se ubicaron las expresiones más dicientes como respuesta a las preguntas especificamente formuladas. Seguidamente se realizó una evaluación detallada de las matrices con el fin de identificar la aparición de las primeras categorías analíticas" (Spiggle, 1994). A partir de la matriz inicial que contenía la pregunta realizada a cada entrevistado, su respuesta -códigos in vivo(Guillemette, 2006) y el análisis correspondiente, se elaboró una nueva matriz con un proceso de precodificación. Luego se elaboró una nueva matriz simplificada que contenía una codificación basada en los análisis de las matrices previas, que permitió describir los hábitos de compra de la población analizada, hasta lograr los niveles de saturación de la información que permitieron concluir el proceso investigativo. Siempre "se buscó leer la realidad a partir de la convergencia entre reflexión, datos del terreno, análisis de contenido, revisión teórica, formulación de aproximaciones teóricas; siempre respetando la rigurosidad y la honestidad intelectual implicada en estos procesos de lecturas subjetivas de complejas realidades" (Páramo, García, \& Arias, 2011). 


\section{RESULTADOS:}

En términos generales, los resultados muestran compradores cuyo comportamiento está signado por la tradición de sus hábitos. Aunque algunos de ellos adquieren sus productos y marcas predilectas a través de canales virtuales, siguen prevaleciendo los patrones aprendidos en la familia y amigos a través de los procesos de socialización del consumo incorporados a sus vidas (Páramo, 2010). La disputa por la preferencia de los compradores es evidente y se refleja en que todos los locales comerciales existentes en la zona cuentan con consumidores que los prefieren por unas u otras razones.

\subsection{Disparador del proceso de decisión de Compra}

Se encontraron 3 aspectos que "disparan" el proceso de adquisición:

a) Consumidor que se percata que hay desabastecimiento total en su hogar, y toma la de decisión de compra de forma inmediata:

"...Cuando ya no hay nada, por ejemplo, eh tratamos de agotar todo al máximo para evitar un sobreabastecimiento porque eso nos pasó en nuestra primera compra como casados, compramos de todo y con el dolor del alma botamos más de la mitad de la compra, cosas que se pudrieron, pensamos que todos los días nos vamos a levantar con ánimo de cocinar, grave error, después de que nos casamos entonces ahora lo que hacemos es programar comida, programar días y a medida que van pasando los días y se van acabando las cosas vamos haciendo la lista de lo que se está acabando y de lo que se va a comprar, pero si esperamos hasta el último momento incluso cuando ya casi no hay nada, para... y más en estos tiempos de ahora" (Entrevista 2). b) Consumidor que tiene una programación consciente para esta actividad con una frecuencia regular preestablecida:

"...yo normalmente lo hacía cada quincena, o sea, que se disparaba era que cada quincena yo lo hago, pero ahora en esta cuarentena me ha tocado hacerlo semanal. Porque siempre hay una necesidad y otra que no se puede suplir si no haría semanal..." (Entrevista 5).

“...normalmente hacemos compra solamente para una semana. Entonces lo hacemos los lunes." (Entrevista 1).

c) Consumidor que observa que sus víveres están disminuyendo, pero no está totalmente desabastecido.

"por lo general él, lo que dispara es cuando la, cuando tú ves que la nevera se está, se está acabando" (Entrevista 3).

"Usualmente porque, que no quiero como quedarme como desabastecida, para, para mis comidas" (Entrevista 8).

Estos aspectos pueden ser considerados como el primer paso para la toma de decisiones que aunque sin ser completamente racionales (Shiffman y Kanuk, 2001), sí tienen un alto componente relacionado con la necesidad de satisfacer las necesidades de supervivencia claramente señaladas por diferentes autores (Páramo y Ramírez, 2017).

\subsection{Influenciadores de la decisión}

Estos inciden directamente tanto en los productos a adquirir como en los sitios a visitar. Su punto de vista, consejo o condición representa un aspecto importante para tener en cuenta por parte del comprador. Se detectaron varios tipos de influenciadores.

\subsubsection{Familia}

Dentro de este grupo de influenciadores están familiares directos del comprador que pueden o no vivir con él, pero que terminan haciendo recomendaciones 
basadas en sus experiencias, gustos, deseos y necesidades. Dentro de los mencionados se encuentran los cónyuge, hijos, madres y cuñados:
“...Estefany (cónyuge) solamente, generalmente ella es la que dice, eh ella es la que lleva la lista, yo le entrego la lista y ella dice falta esto, falta lo otro, faltan manzanas, falta apio, falta esto. $Y$ a veces ella dice: ay quiero... vamos a agregar esto dulce un capricho, algo así que no está en la lista pero que en su momento pues se antojó". (Entrevista 2).
"bueno por lo menos aquí... influyen por 10 menos los dos, porque por lo menos Gerardo mi esposo, él no puede comer tanta azúcar. $Y$ la niña también esta como en esa cuestión de mami: no compres tanto dulces, ya no compres tantas galletas, trata de comprar siempre más como cosas integrales y más frutas y verduras" (Entrevista 3).

Convertida en la célula básica de la sociedad se reitera el papel jugado por la familia no solo en el aprendizaje de los hábitos de consumo prevalecientes en determinada cultura, sino que inciden de manera directa en la decisión de consumo y de compra (Páramo, 2010).

\subsubsection{Redes sociales}

Otros influenciadores pertenecen a las redes sociales, que últimamente se han vuelto muy importantes para impulsar los estilos de vida saludable que terminan impactando la decisión de compra.

"yo por ejemplo me guio de cosas de comida Fit. Entonces cuando veo que recomiendan algún nuevo producto 0 alimento o algún tipo nuevo de verdura, ehhh voy y buscó para ver cuáles hay. Por lo general en el Carulla lo encuentro. Entonces voy lo pruebo y miro para ver qué tal, le miró los ingredientes como te dije. Entonces sí... digamos que adicional a nosotros ehhh algo más que puede influenciar la escogencia de mis productos, es influenciadores de... de las redes sociales" (Entrevista 1).

\subsubsection{Personal Especializado en salud (Nutricionista)}

Debido a las nuevas tendencias en alimentación saludable, este tipo de influenciador, que es más especializado, termina incidiendo en los productos alimenticios que se adquieren y por ende también en el lugar donde se adquieren dichos productos.

"...la influencia más reciente como de las cosas que llevo comprando hace más o menos un año o más, pues mi nutricionista, que ella si me daba como una lista del mercado y me decía compra esta marca, que esta marca tienen más azúcar, esta tiene menos carbohidratos, tal, ella es como la influencia más grande recientemente como en mí, en mi manera de escoger los productos" (Entrevista 9)

\subsubsection{Amigos}

Otro grupo de influencia son los amigos; personas cercanas al comprador que debido a su experiencia terminan haciendo recomendaciones que son atendidas dada su cercanía afectiva y a la credibilidad que se les tiene:

"Amigos que nos dicen: no mira que los detergentes de Ara, que son muy buenos, que eso y ya hemos probado y bueno si en algún momento." (Entrevista 4)

"...ehh, con mis amigas y con mis compañeros de trabajo también compartimos bastante cosas de la casa, entonces." (Entrevista 6).

Los amigos como grupo de referencia claves en el proceso de socialización del consumo se convierten en notables influenciadores en el proceso de decisión de compra (Páramo y Ramírez, 2019)

\subsection{Factores que influyen en la selección del sitio dónde realizar la compra.}

Los resultados muestran dos tipos de categorías que fueron agrupadas en dos factores distintos aunque complementarios entre sí. Unos asociados a la percepción de los compradores y, otros, relacionados con el servicio prestado por cada local. 


\subsubsection{Aspectos de percepción.}

\section{a) Higiene}

Algunos entrevistados manifiestan que la higiene percibida es un factor fundamental al decidir dónde hacer la compra; consideran que el lugar debe verse organizado, limpio y agradable. Si el lugar no cuenta con esas características, podría ser descartado:

"...que se vea agradable, que no vayan a tener olores..." (Entrevista 9)

"aparte de que me quede cerca que me, que yo encuentre todo, bien organizado, limpio" (Entrevista 11).

\section{b) Calidad de los productos.}

Los entrevistados mencionan que la calidad percibida de los productos es un factor decisivo para escoger el sitio. Si piensan que los víveres son de mala calidad o existe un riesgo de intoxicación el local comercial es descartado:

"Carulla me gusta es la calidad por decir, de las carnes y los productos como los maneja, como, como más higiene, en cambio uno va a la Olímpica y siempre las carnes huelen como un olor feo yo no sé yo siento un olor como feo, en las carnes, como en los pollos, el pollo no me gusta, tiene, mal sabor y yo no soy la única que lo dice" (Entrevista 3)

\section{c) Amabilidad de los empleados}

Algunos entrevistados mencionaron que el buen servicio -asociado a la buena atención- es determinante a la hora de escoger un supermercado, incluso tienen en cuenta la hora cuando consideran que entre más temprano se haga la compra serán mejor atendidos, debido a que los empleados no estarán tan cansados. Todo en concordancia con los investigado por Páramo y Ramírez, (2007):

"me atienden como mejor en la mañana están como frescos ya como a la 1 de la tarde veo que ya la gente cansada y entonces siempre miro como que eso en la mañana está entrando un turno apenas esa persona esta cómo con esas amabilidades ya la 1:00 de la tarde esta persona y está cansada" (Entrevista 3).

\section{d) Apariencia de los alrededores}

Para algunos entrevistados la apariencia de los alrededores es un factor importante al momento de elegir el lugar en donde hacer la compra. Si la ubicación no es percibida como segura, es descartada con el fin de no exponerse a un posible robo:

“...entonces, la otra Olímpica está llena como de Mototaxis en la puerta, gente, ay, llega muchísima más gente, porque es una Olímpica que está más visible y queda como en, ehh, en una zona donde hay mucha más población, queda alli al lado de la Playa y aquí en Villa Campestre, la otra está como más escondidita, va menos gente, entonces uno hace más tranquilo la compra" (Entrevista 8)

\section{e) Comodidad}

Para algunos entrevistados la comodidad y facilidad para adquirir los productos es importante e influye en su decisión de compra, ratificando lo que encontró Páramo, (2009) en su estudio pionero de las tiendas de barrio como reforzadoras de la identidad del colombiano:

"Definitivamente y comodidad, facilidad." (Entrevista 11)

\section{f) Sensación de estatus}

Algunos entrevistados mencionaron que prefieren hacer el mercado en un mismo lugar, así deban invertir un poco más de dinero, esto por razones de tiempo y comodidad (Páramo, 2009; 2010).

"Entonces ir, ir a un lado y después ir al otro y después ir al otro, no, yo saco todo el mismo lunes en Carulla sin importar si cuesta un poquito más. Gente pupi. (Risas)". (Entrevista 1) 


\section{g) Seguridad}

Algunos entrevistados eligen el sitio de compra teniendo en cuenta la seguridad percibida, sobre todo para aquellos que van acompañados de sus hijos. Si perciben inseguridad dentro del establecimiento, lo descartan como opción (Páramo, 2009).

"Yo por ejemplo defiendo Carulla a morir por el asunto de la seguridad de las niñas." (Entrevista 1)

\section{h) Presentación de los productos, estantería agradable y colores}

Múltiples entrevistados manifestaron la importancia de la presentación de los productos y la organización de la estantería como factores determinantes para seleccionar el sitio donde realizar la compra de sus productos. Mencionaron que inclusive los colores son importantes para tener una sensación agradable del lugar de compra:

"...Es un lugar desordenado que tu llegas y tú no encuentras la verdurita bien puesta y eso, $y$ en eso si tú vas a hacer un, una compra presencial a mi si me afecta y a mi esposo peor, o sea, el acostumbrado a su Carulla a su lugar espectacular, entonces nosotros por ejemplo Ara nunca hemos logrado a pesar que tiene buenísimos precios y que tiene unas promociones; mi hermana me dice compre no sé cuántas cosas y yo, y me costó tanto y yo pero baratísimo, y así, si fue que entre a Ara, y yo ah no, pero es que yo no entro a Ara me parece desordenado y me desespera el no, que no ver que esta todo bien delineadito" (Entrevista 9).

\section{i) Tamaño del supermercado}

Para algunos entrevistados el tamaño del supermercado es un factor decisivo al momento de escoger el lugar de la compra. Prefieren supermercados pequeños, pero donde encuentren los productos que necesitan (Páramo, 2009):

"Desde que nacieron mis hijas eh procuro, 0 sea Lucía tiene 11 años ya, procuro ir a, así a supermercados pequeñitos para poder estar pendiente. Antes que me mudará aquí a Villa Campestre lo hacía en la Olímpica de Miramar que también es pequeñita" (Entrevista 9)

\section{j) Rapidez en la atención}

Para algunos entrevistados la rapidez en la atención es fundamental al momento de escoger un lugar para mercar. Son personas que tienen poco tiempo para invertir en esta actividad y que valoran la agilidad en el servicio (Páramo y Ramírez, 2007).

"y como es rápido, me gusta que me atienden rápido, digo que yo a las horas que yo llego eh, de pronto es eso también que yo ya sé que hay horas en las que no debo ir, entonces a la hora que yo llego, todo lo puedo, para mi el tiempo es valioso y ahora pues, también no me gusta estar mucho tiempo en, en lugares cerrados, con mucha gente entonces para mí el tiempo es muy valioso" (Entrevista 10)

\section{k) Cantidad de clientes en el supermercado}

Algunos consumidores indicaron que prefieren lugares con pocas personas a la hora de hacer el mercado, porque así se pueden movilizar fácilmente, requieren poco tiempo y pueden salir rápidamente del establecimiento:

"...desde antes de la pandemia a mí me daba como estrés tener el poco de gente y que sea como algo como tan pequeño y uno con el poco de gente antes de que pasara esto nunca me ha gustado así, entonces siempre iba ahora que... como te digo, a almacenes que no estuvieron tan llenos, que fueran como fácil de yo de salir de ellos, de no, de no estar alli tanto tiempo" (Entrevista 3)

\section{I) Disponibilidad de marcas}

Para algunos, la marca de los productos que se encuentran en el supermercado 0 incluso la misma marca del supermercado, es relevante al momento de escoger donde hacer el mercado. Hay compradores aparentemente fieles a ciertos productos y/o establecimientos:

"lo que es Justo y Bueno, D1 y Ara, hay cosas que no tiene, y no tienen todas las marcas, a 
HÁBITOS DE COMPRA DE ALIMENTOS DE LAS FAMILIAS RESIDENTES EN LA ZONA DE VILLA CAMPESTRE DE BARRANQUILLA, COLOMBIA Carlos Andrés Ortiz Coronado - Dagoberto Páramo Morales

veces marcas que uno no conoce, eh, o simplemente no las tienen, productos que no tienen, no manejan" (Entrevista 6)

"...Yo tengo eso en mi mentalidad de la Olímpica siempre...Yo me relaciono con la marca, es eso, es un tema relacional de toda la vida con la marca.... Eh, y en realidad siento que la Olímpica es mía (Risas) es de la Barranquilla" (Entrevista 5)

\subsubsection{Aspectos relacionados con el negocio}

\section{a) Variedad y Características de los productos}

La variedad de productos es un factor importante para ciertos entrevistados. Los compradores prefieren un negocio que tenga todos los productos requeridos para no tener que desplazarse entre varios establecimientos:

"que encuentre pues variedad también de cosas que no me toque estarme desplazándome de un supermercado para otro porque no encuentro que aquí nada más encuentro unas cositas y me toca irme para otro no" (Entrevista 11)

\section{b) Relación calidad-precio}

La relación calidad-precio influye en algunos consumidores. En algunos productos, en especial los de alimentación, ellos buscan que sea de buena calidad, que no deterioren su salud, pero también analizan que el precio sea justo.

"Si influye, pero de pronto para determinados productos para mí. O sea, de pronto si tú me dices que no sé, que una servilleta más barata, más económica, ehh, un jabón de platos más económico, ehh, cosas que de pronto, pero cuando ya entra, ah eh, a ser un producto relacionado con mi salud o con qué me alimenté si, si somos más, buscamos un balance de costo-beneficio, no necesariamente va a ser lo más económico lo que vamos a comprar, cuando es ya de consumo de, de, para alimentarnos, no" (Entrevista 6)

\section{c) Precio}

Sólo el precio de los productos a comprar es la motivación principal de algunas personas para decidir hacer el mercado en cierto establecimiento:

"Yo en realidad la mayoría de las veces me decido es por precio en realidad. Por precio, algunitos productos es porque me gustan. Algunos productos porque me gustan, pero la mayoría es por precio" (Entrevista 5).

\section{d) Ubicación y cercanía}

La ubicación y cercanía son otro aspecto importante para algunas personas que influyen en su decisión de compra:

"Cuando íbamos presencialmente. Eh, eh, bueno, Price Smart porque nos queda cerca, y además porque hay unos productos que nos gustan bastante por su calidad, no todo 10 comprábamos ahí, por ejemplo, ahí no comprábamos tanto las verduras" (Entrevista 7)

\section{e) Promociones de ventas}

Las promociones son otro aspecto importante, prefieren lugares que ofrezcan descuentos especiales, ya sea por el medio de pago o por otras razones:

"no tienes en realidad para comparar y mirar y mirar que hay en promoción y que pusieron a veces en Carulla colocan como activa este cupón con, por la aplicación de Carulla y cuando pagues te haremos el descuento. Entonces son cosas que, que te pierdes cuando lo haces por la aplicación" (Entrevista 1)

\section{f) Preferencia de marca}

Hay entrevistados que mencionaron que prefieren un supermercado porque encuentran las marcas y productos de su preferencia:

"...o por ejemplo me guio de cosas de comida Fit. Entonces cuando veo que recomiendan algún nuevo producto o alimento o algún tipo nuevo de verdura, ehhh voy y busco para ver 
cuáles hay. Por lo general en el Carulla 10 encuentro" (Entrevista 1)

\section{g) Servicio a Domicilio}

La rapidez, facilidad y cercanía de los establecimientos son un factor importante para ciertas personas a la hora de solicitar el servicio a domicilio. Si el establecimiento queda lejos de su residencia, consideran que el tiempo de espera para que lleguen los productos puede ser muy alto.

\begin{abstract}
"Sí. Facilidad, cercanía, rapidez también ahora en temas de domicilio, pues es más fácil que te llegue un domicilio de la Olímpica que está aquí mismo que de pronto pedir en un Jumbo o algo que no, que no va a llegar tan, tan rápido, tan inmediato, entonces en estos, ya cuando son productos si muy especiales que no, yo compro la carne en tal lado, entonces si trato de acumular y hacer un solo pedido para que me alcance, pero lo que es temas de supermercado de superficies, si los hago en Villa Campestre" (Entrevista 6)
\end{abstract}

\subsection{Tipología de consumidores}

Dentro de la investigación se indagó sobre las razones por las cuales la persona entrevistada era la encargada de realizar la compra de los víveres, así como el canal de compra preferido. Basados en estos factores diferenciales emergió una tipología de consumidores desde diferentes perspectivas (Spiggle, 1994):

\subsubsection{Tipos de consumidores por asignación de hacer la compra}

\section{a) Organizado}

Este tipo de consumidor se considera el más organizado en el hogar para comprar:

"yo soy más ordenada y compro menos (risa Nerviosa). O sea, por lo menos de pronto si yo lo mando con una lista créeme que va a traer todo lo contrario de la lista, va a traer el doble de las cosas" (Entrevista 9)

\section{b) Chef del Hogar}

Este tipo de consumidor es el encargado de preparar alimentos.
"Y, bueno yo cocino. Yo cocino entonces como es más fácil planificar lo que voy a necesitar $y, y$ lo que vamos a consumir, entonces por ese lado eso, y la otra es que, por el tema de la comida yo trato de, de que sea, no es que, sea obsesiva con el tema de lo saludable ni nada, pero si, si me gusta comer bien, mi esposo es más goloso, entonces trato de yo planificar la, la, la compra" (Entrevista 6)

\section{c) Administrador}

Este tipo de consumidor es el encargado de administrar presupuestos en el hogar.

"Siempre junto con, con mi esposa. Bueno porque yo llevo la plata (Risa)" (Entrevista 7)

\section{d) Por iniciativa propia}

Este tipo de consumidor que simplemente escogió ser quien compra en su hogar.

"Pues, porque tomo la iniciativa (risas) cuando la nevera está vacía, cuando ya veo que se están acabando las cosas, entonces no espero a que no haya nada, al día siguiente voy yo y salgo a hacer el mercado." (Entrevista 8)

\subsubsection{Tipos de consumidor de acuerdo con el canal de venta preferido}

Dentro de la investigación se encontraron varias características de los consumidores que permitieron clasificarlos por el canal que utilizan para realizar las compras.

\section{a) Consumidor Presencial}

Realiza el $100 \%$ de sus compras de manera presencial, Compara productos (calidad y precio). Revisa promociones. Prefiere mirar, palpar, comparar. Este tipo de consumidor manifiesta también realizar compras por impulso en el momento de la compra. Las ventajas más relevantes que ven están asociadas a que este canal es percibido como más económico ya que es posible comparar qué conviene en cuanto a cantidades, tamaños y calidades. En contraste, se señala que su principal desventaja está relacionada con el tiempo requerido para recorrer 
varios negocios y el mayor riesgo de contagio por cuenta de la pandemia que se está padeciendo.

Dentro de este tipo de consumidor presencial se encuentran varios subtipos que fueron clasificados así:

- Planificador: Va con la lista en mano y selecciona los productos que necesita, se programa previamente.

- Especialista: Sólo ingresa a áreas específicas para determinados productos, ejemplo productos saludables, zona de verduras.

- Explorador: Recorre todos los pasillos, sea como estén dispuestos por el supermercado 0 de forma aleatoria.

- Solitario: Este tipo de comprador prefiere realizar las compras sólo. Las razones indicadas para esta preferencia son: tranquilidad para no sentir presión del acompañante, rapidez para terminar en menor tiempo la actividad y, reducción de costos ya que cuando se va acompañado es probable gastar más.

- Acompañado: Por el contrario, otro grupo de entrevistados, -la mayoría-, prefiere realizar el proceso de compra en compañía. Las razones encontradas se asocian al reparto de actividades: dividirse, ayudarse a escoger, pesar, agregar productos al carrito, hacerlo más eficiente y rápido.

\section{b) Consumidor virtual situacional}

Debido a la situación actual de pandemia, varios consumidores usan medios virtuales, se sienten seguros, y tienen menos riesgo de contagio. Se encontró también presente el componente de compra por impulso en este tipo de consumidores.

"Eh, bueno ahora mismo se está pidiendo todo a domicilio, este... Se pide, investigamos primero por internet donde están las ofertas, eh, también dependiendo de, influye bastante también cuanto cobran por el domicilio, eh, influye también bastante si se demoran mucho en, en traer el domicilio" (Entrevista 7)

"Bueno en este momento lo que me motiva es que no me parece seguro ir al supermercado. O sea, en el supermercado la gente toca todo, eh, hay más, o sea, hay probabilidad de contagiarse, entonces eso me parece, un, un peligro que puedo evitarme" (Entrevista 8)

Para la realización de la compra se usan aplicaciones móviles como Rappi, la de Olímpica, Whatsapp, llamadas telefónicas a tiendas especializadas y aplicaciones de otros supermercados.

Entre las ventajas que tienen al acudir a canales virtuales indican que no solo obtienen los mismos descuentos presenciales, sino que sienten que pagar el costo del domicilio es equivalente al costo de la gasolina para transportarse. Mencionan, igualmente, que al ser virtual y usar una lista no hay margen para hacer compras inesperadas -por impulso- en el punto de ventas. Comentaron también que al comprar en varios lugares les es posible obtener un ahorro de tiempo y de dinero en transporte. Adicionalmente, pueden tener a alguien que desde el supermercado les avise si hay disponibilidad del producto pedido y en caso contrario, les ofrezca productos sustitutos.

Entre las desventajas, algunos perciben estas compras virtuales como más costosas. Otros manifiestan que se reduce la libertad de escoger determinado producto puesto que este proceso está sometido a la decisión de la persona que les empaca el mercado. Varios entrevistados indicaron que prefieren escoger el producto presencialmente, viéndolo, tocándolo, oliéndolo; consideran que es una desventaja el no poder comparar tamaños, calidades, frescura y cantidades de la misma forma que presencial. Mencionan que no pueden conocer las promociones disponibles en el supermercado. También manifiestan sentir incertidumbre de lo que les van a enviar, o que el producto que les envíen no sea el que se pidió. En ocasiones no pueden encontrar todos los productos que requieren y que aparecen en la aplicación, sino que debe buscarlos en varias páginas de productos. Algunos no se sienten cómodos con el no poder interactuar con el producto, y poder revisar, además del precio, las especificaciones técnicas. Sienten poca libertad para escoger productos. 


\section{c) Consumidor Mixto}

Este tipo de consumidor utiliza medio presencial y virtual incluso previo a la pandemia:

"Me ha gustado lo, lo virtual, me ha gustado hacer los pedidos y todo, no me ha disgustado. En realidad. De pronto 10 seguiría. Lo seguiría haciendo, sí. De vez en cuando, yo voy al supermercado y voy debes en cuando a Ara por lo menos voy, porque a Ara yo me siento un poquito más segura, porque siento que es de menos gente y de menos. Entonces de pronto son las 8:00am y voy un momentico y saco lo que tengo que comprar, pero a la Olímpica como sé que voy a comprar más prefiero, el domicilio. Me ha ido bien, me fue mal por la página de ellos. Por la aplicación, pero me fue bien por el WhatsApp" (Entrevista 5)

\subsubsection{Número de sitios comerciales visitados}

\section{a) Un solo supermercado:}

Existe un tipo de consumidor que prefiere realizar sus compras en un solo lugar. La razón expresada es que siempre encuentra los productos que requiere. No le gusta visitar varios sitios porque le toma más tiempo a menos que sea a domicilio.

"Me gusta hacer compra en uno solo, entonces no soy muy, y ya me acostumbré así, por lo menos me han Salido tú sabes que estamos en Villa Campestre, nos sale Justo y Bueno y el otro que nos salió y yo me he quedado todavía en Ara porque ya estoy acostumbrada a comprar en Ara" Entrevista 5

\section{b) Diferentes supermercados:}

Existe un tipo de consumidor que indica que acude a diferentes supermercados y tiendas ya que compra dependiendo del producto en tiendas especializadas 0 en otros, incluso, en espacios públicos.

"Porque hay cosas que me gusta comprarlas donde es, son especialistas en eso. Como te decía, eh, me parece que el pescado a mí me parece más fresco en una pescadería que si lo compro en un almacén donde venden pescado, carne y pollo. La carne también me gusta comprarla fresca en un almacén especializado en carnes y el pollo también, eso, y lo que es verdura aparte y ya. Varios puntos, tal cual, yo iba a varios puntos y a veces como me tocaba, eh, días diferentes entonces de pronto un sábado iba a uno, otro sábado iba a otro..." (Entrevista 6)

\subsubsection{Horarios preferidos}

Existen dentro de los consumidores de la zona dos horarios preferidos para realizar el proceso de compra de sus víveres:

\section{a) Matutino (7AM a 12M):}

Las razones encontradas para escoger este horario es la "frescura" de la hora, más cómodo, no está tan lleno, encuentran una mejor atención ya que el personal está descansado, encuentran productos más frescos, las instalaciones están más limpias y hay un mejor abastecimiento.

\section{b) Vespertino (6PM a 7PM):}

Este tipo de horario es preferido por consumidores que deben cumplir un horario laboral y por tanto realizan sus compras al salir del empleo y dirigirse a sus hogares.

\subsubsection{Días preferidos}

Se encuentra que existe dentro de los consumidores de la zona unos días particulares de la semana para realizar la actividad de comprar sus víveres.

\section{a) Miércoles:}

Dentro de las razones encontradas es que es un día de descuento en la Olímpica, uno de los supermercados más frecuentados.

\section{b) Lunes:}

Dentro de las razones encontradas es que prefieren este día para poder planificar la alimentación de la semana

\section{c) Martes:}

Prefieren este día ya que hay descuento en Carulla uno de los supermercados más frecuentados.

\section{d) Fines de semana (sábado y domingo)}


Dentro de las razones mencionadas están el poder tener tiempo para organizar, desinfectar y almacenar los productos; una actividad que, según se argumenta, resulta agotadora por el tiempo que toma. Hacerlo entre semana en la noche implica un esfuerzo adicional luego del trabajo. Indican que antes de la pandemia aprovechan el fin de semana para pasear y distraerse, compartiendo con la familia.

\section{e) Según conveniencia/sin un día preferido:}

También se encontró que existe un tipo de consumidor que no realiza la compra un día en particular, no tiene un día preferido, sino que lo hace según su conveniencia o necesidad. Este consumidor no compra en días de descuento porque es probable que en el lugar existan muchas filas, muchas personas y resulte una experiencia muy "caótica". Prefiere la comodidad a ahorrarse unos pesos. No presta atención a los días de descuento; de hecho, algunos manifiestan no creer en esos días 0 , dudan que los productos tengan un descuento real y prefieren realizar esta actividad según la conveniencia de su tiempo.

\subsubsection{Frecuencia de compra}

Fueron encontrados 3 frecuencias de compra dentro de los consumidores entrevistados: cada 8010 días, cada 15020 días y, cada 30 días

Las principales razones para realizar la compra en estas frecuencias son: la caducidad de alimentos perecederos, la duración de los elementos de aseo evitan el deterioro de los productos-, no comprar en exceso, -nevera pequeña-, no tener vehículo, la situación actual de pandemia, tener un mejor control del consumo, por organización, por sentirse abastecido más tiempo sin pensar en tener que comprar y, para poder pagar todo de contado con su ingreso que también tiene frecuencia quincenal.

\subsubsection{Medios de pago preferidos}

\section{a) Tarjeta débito:}

El medio de pago más utilizado es la tarjeta débito dado que, en general, los consumidores no manejan efectivo -por seguridad y comodidad-, no le gusta manejar tarjeta de crédito ya que la relacionan con endeudarse y no poder pagar. Les parece más práctico el uso de tarjeta débito.

\section{b) Tarjeta de crédito:}

Se encontraron consumidores que prefieren este medio ya que pueden obtener beneficios financieros pagando a 1 cuota lo que no les genera intereses, pueden acumular millas y mejorar su historia crediticia. También fue mencionado el uso de tarjeta de crédito de la marca Olímpica la cual permite acceder a descuentos adicionales y mejorar el control de pagos al hacerlo a través de un solo medio.

\subsubsection{Duración del proceso de compra}

Dependiendo de si el proceso de compra se realiza de forma presencial o virtual la duración del proceso es variable.

\section{a) Compras presenciales}

De forma presencial fue mencionado que puede demorar entre 1 a 1.5 horas en unos casos y entre 2 y 3 horas en otro. Las razones se asocian a la cantidad de cajeros disponibles en el supermercado, a la duración de las filas para pagos, a la revisión de los precios y las fechas de vencimiento por parte de los compradores, a la distancia física que debe tomarse por la pandemia, a recorrer todos los pasillos de los supermercados, a observar otros productos que no necesariamente van a ser comprados, y, a la búsqueda de algunas marcas particulares.

\section{b) Compras virtuales.}

De forma no presencial se requiere una menor cantidad de tiempo para realizar las compras. Fueron mencionados los rangos entre 10 y 15 minutos, entre 30 y 40 minutos y hasta una hora. Las razones para que tome este tiempo son el pedir a domicilio y dictar la lista de artículos 0 escogiéndolo dentro de las aplicaciones.

Fue mencionado en ambos casos que debido a las recomendaciones dadas en la situación actual de pandemia, deben agregar al tiempo del proceso de compra, un periodo de lavado de los productos que consume varias horas. 


\section{CONCLUSIONES:}

En el entorno de la zona estudiada con presencia de un número creciente de supermercados en constante competencia por captar a sus clientes, se detectaron varios factores que tipifican los hábitos de quienes realizan las compras de los productos de la canasta de alimentos. Se encuentran varios tipos de consumidores. Unos que prefieren los medios virtuales bastante influenciados por las condiciones actuales de la pandemia de Covid 19 con una fuerte tendencia a la utilización de los servicios a domicilio. Otros que, por el contrario, manifiestan preferir, para este tipo de compras, realizarlas de forma presencial para poder relacionarse mejor con el producto a través de no sólo el sentido de la vista como lo hace un comprador virtual sino usando varios de sus sentidos. Es evidente en las conversaciones que muchos fueron forzados a usar otros canales diferentes a las compras presenciales lo cual obligó a muchos supermercados y a tiendas especializadas a buscar otros medios para poder satisfacer necesidades de sus usuarios. Es por eso por lo que mencionaron el uso de Whatsapp, redes sociales, aplicaciones móviles, el uso del teléfono para realizar la compra de los víveres. Son varios los aspectos que tienen en cuenta los compradores para realizar el proceso de abastecerse, desde el canal que utilizan, la frecuencia con que lo hacen, aspectos de percepción sobre los lugares y productos como limpieza, olores, colores, las recomendaciones de familiares y amigos.

Existe tendencia a realizar compras en mayor medida en 2 supermercados de la zona, Olímpica y Carulla, los más conocidos y los de mayor tradición. Sin embargo, fue evidente la presencia en los comentarios de las tiendas Hard Discount lo cual comprueba la vigencia y penetración de este tipo de negocios aun en zonas de estratos 4,5 y 6 , que son los que predominan en el área. Se evidencia una fuerte tendencia en los entrevistados a pagar con tarjeta débito dejando a un lado el dinero en efectivo.

Fue bastante mencionada la dificultad para realizar el lavado y desinfección de los productos. Varios entrevistados indicaron con desagrado que por la pandemia esto ahora hace parte de su proceso de abastecerse. Se referían en mayor medida al tiempo que toma esta actividad particular para luego organizar sus provisiones.
Se encuentra un grupo bastante heterogéneo de compradores en cuanto a gustos y hábitos lo cual es bueno para realizar posibles campañas de mercadeo segmentadas.

A pesar de tener gran variedad de supermercados en la zona, existen compradores que prefieren tiendas especializadas fuera de la zona. El uso del domicilio se ha incrementado de forma sensible por la situación de pandemia.

Podría pensarse que esta zona pudiera estar saturada por los comercios existentes, sin embargo, es posible hacer un estudio de mercado más especializado para evaluar la posibilidad de tiendas especializadas como en alimentos fit o pescadería y mariscos.

De todos los productos mencionados se observa que existe una tendencia en los entrevistados a preocuparse por mantener un estilo de vida saludable, respaldado en su alimentación, observándose un rechazo al azúcar y a los dulces. También a realizar la compra en las mañanas y en casi todos los días de la semana, ninguno mencionó el jueves como día escogido para la actividad. Hay influencia de los días de descuento de los supermercados en varios casos, sin embargo, hay compradores que no prestan atención a estos días y prefieren hacerlo según su conveniencia y disponibilidad de tiempo

Si bien parte de los entrevistados ha usado medios virtuales, no se observó que estuvieran completamente satisfechos con el servicio ya que mencionan diferencias entre lo requerido por medios virtuales y lo que finalmente reciben en sus hogares a domicilio. Esto puede ser aprovechado para mejorar los medios digitales actuales de los supermercados o buscar un espacio para un nuevo "jugador" intermediario en este tipo de venta, que pueda aprovechar esa dificultad para iniciar un emprendimiento.

\section{REFERENCIAS:}

Allen, A., da Silva, N. L., \& Corubolo, E. (03 de 1999). Obtenido de ENVIRONMENTAL PROBLEMS AND OPPORTUNITIES OF THE PERIURBAN INTERFACE AND THEIR IMPACT UPON THE POOR: https://discovery.ucl.ac.uk/id/eprint/37/1/DPU _PUI_Allen_Corubolo_daSilva_Environment al.pdf 
Allport, G.W.(1961). Pattern and Growth in Personality. New York: Holt, Rinehart and Winston.

Andrade, C. (12 de 03 de 2019a). ¿Qué compran los colombianos según su generación? Obtenido de

https://www.kantarworldpanel.com/co/Noticia s/Qu-compran-los-colombianos-segn-sugeneracin

Ardila, E., \& Rueda, J. (30 de 09 de 2013). La saturación teórica en la teoría fundamentada: su de-limitación en el análisis de trayectorias de vida de víctimas del desplazamiento forzado en Colombia*. Obtenido de La saturación teórica en la teoría fundamentada: su de-limitación en el análisis de trayectorias de vida de víctimas del desplazamiento forzado en Colombia*: https://revistas.unal.edu.co/index.php/recs/ar ticle/view/41641/43310

Caro, J. (04 de 03 de 2019). El cambio de los canales de compra en Colombia. Obtenido de https://www.kantarworldpanel.com/co/Noticia s/El-cambio-de-los-canales-de-compra-enColombia-

Contreras, M., Páramo, D., Rojano, Y. (2019). La teoría fundamentada como metodología de construcción teórica. Revista Pensamiento \& Gestión. No. 47. 283-306

Departamento Administrativo Nacional de Estadística. (2018a). Encuesta Nacional de Presupuestos de los Hogares - ENPH. Obtenido de https://www.dane.gov.co/files/investigaciones /boletines/enph/ciudades-enph-2017.xls

Departamento Administrativo Nacional de Estadística. (2018b). Informe de resultados Encuesta Nacional de Presupuestos de los Hogares. Obtenido de https://www.dane.gov.co/files/investigaciones /boletines/enph/presentacion-enph-2017.pdf

Departamento Administrativo Nacional de Estadística. (n.d.). Dane. Obtenido de Conceptos Basicos: https://www.dane.gov.co/files/inf_geo/4Ge_C onceptosBasicos.pdf

Flores, M. (4 de 10 de 2016). En 2030, los Millennials serán el $75 \%$ de la fuerza de trabajo a nivel global: Manpower Group. Obtenido de https://laureatecomunicacion.com/prensa/en-2030-losmillennials-seran-el-75-de-la-fuerza-detrabajo-a-nivel-global-manpower-group/

Fujitsu. (25 de 01 de 2018). Los millennials serán más del $50 \%$ de la fuerza laboral en 2025 y redefinirán la cultura corporativa, según Fujitsu. . Obtenido de https://www.europapress.es/portaltic/sector/n oticia-millennials-seran-mas-50-fuerzalaboral-2025-redefiniran-cultura-corporativafujitsu-20180125151635.html

Guillemette, F. (2006). L'approche de la Grounded Theory; pour innover? . Recherches qualitatives, 26 (1), 32-50.

Jiménez, L. M. (28 de 10 de 2018). Obtenido de https://www.elheraldo.co/barranquilla/habitan tes-del-corredor-universitario-exigenredisenar-tramo-de-la-nueva-circunvalar

McDaniel, C., \& Gates, R. (1999). Investigación de mercados contemporánea. México, D.F: International Thomson.

Páramo, D. y Ramírez. E. (2019) Marketing y cultura: Antropología de consumo. En: Cerda, Luis Manuel y Ramírez, Margarita (Coordinadores), Fundamentos para un nuevo marketing. Editorial Sindéresis. Madrid. pp: 145-170

Páramo, D., García, O., \& Arias, M. (6 de 2011). Hacia una tipología de tenderos de Manizales (Colombia). Obtenido de http://rcientificas.uninorte.edu.co/index.php/p ensamiento/article/viewFile/2250/1466

Páramo, D. (2010) Cultura y consumo, una reflexión crítica. En: Sahui, José (Ed). Mercadotecnia y sociedad de consumo. Fontamara, Colección Argumentos. México. P. 55-97

Páramo, D. y Ramírez, E. (2007) Gerencia Estratégica de Marketing: Un enfoque cultural. Ediciones USCO Universidad Surcolombiana de Neiva.

Páramo, D. (2009). Le commerce traditionnel colombien: un espace ee renforcement 
culturel pour les consommateurs. (Issue 705). Université De Genéve.

Páramo, D. (2004b) Fenómeno de consumo y consumo en marketing. Revista Convergencia 11(34), 405-435.

Páramo, D. (2004a). Marketing, su esencia conceptual. Colombia:Ediciones Uninorte.

Páramo, D. (1999) Hacia un modelo simbólico-cultural del comportamiento del consumidor. Revista Pensamiento y Gestión No. 7, 49-84.

Rodríguez, H. (13 de 03 de 2017). El canal moderno y los mercados emergentes. Obtenido de https://www.esan.edu.pe/conexion/actualidad /2017/03/13/el-canal-moderno-y-losmercados-emergentes/

Schiffman, L., KanuK, L. (2001). Comportamiento del Consumidor. México: Prentice Hall (7ta edición).

Spiggle, S. (1994). Analysis and Interpretation of Qualitative Data in Consumer Research, Journal of Consumer Research 21 (Dec), 3.

Stanton, W. J., Etzel, M. J., \& Walker, B. J. (2007). Fundamentos de Marketing. México: MacGraw-Hill.

Solomon, M. R. (1997). Comportamiento del consumidor. México: Prentice Hall (3ed.)

Strauss, A., \& Corbin, J. (1990). Basics of Qualitative Research: Grounded Theory Procedures and Techniques. Beverly Hills, C.A: Sage Publications.

Valencia, D., \& López, F. (13 de 10 de 2013). ZONAS SUBURBANAS. Obtenido de http://www.scielo.org.co/pdf/agor/v14n1/v14n 1a04.pdf 